\title{
A Frequency-responsive Photoelectric Design for Highly Sensitive Photoelectrochemical Response Measurements
}

\author{
Jian Wang ${ }^{1}$, Liping $D u^{1}$, Wei Chen ${ }^{1}$, Ping Wang ${ }^{2}$, Chunsheng W ${ }^{1 *}$ \\ 1 Institute of Medical Engineering, School of Basic Medical Science, Health Science Center, Xi'an \\ Jiaotong University, Xi'an 710061, China \\ ${ }^{2}$ Biosensor National Special Laboratory, Key Laboratory of Biomedical Engineering of Ministry of \\ Education, Department of Biomedical Engineering, Zhejiang University, Hangzhou 310027, China \\ ${ }^{*}$ Correspondence: C. Wu (e-mail: wuchunsheng@xitu.edu.cn)
}

\begin{abstract}
A new design derived from light-addressable potentiometric sensors (LAPS) and light-activated electrochemistry (LAE) for highly sensitive photoelectrochemical sensing has been proposed. In this system, a bias voltage is applied to create a depletion layer at the semiconductor/electrolyte interface. A modulated light illuminates the sensor structure to generate electron/hole pairs and causes a detectable alternating photocurrent. The results showed a much higher signal-to-noise ratio (SNR) of the photoelectrochemical response of silicon substrates compared to that using the traditional linear sweep voltammetry (LSV) measurement. Moreover, a pH sensitivity of about $36 \mathrm{mV} / \mathrm{pH}$ was obtained analogous to LAPS with a $\mathrm{SiO}_{2} / \mathrm{Si} /$ electrolyte sandwich structure, but with a steeper photocurrentvoltage curve due to the absence of the insulator layer. The proposed simple and low-cost sensor system can not only be used for photoelectrochemical response measurements of different semiconductors with high SNR, but also be prospective for the sensing of ions, DNA or living cells with spatial reoslution.
\end{abstract}

Key words: Photoelectrochemical, frequency-responsive, high sensitivity, biosensor

\section{Introduction}

The investigation of photoelectrochemical behaviour of semiconductors to convert irradiative energy to electrical or chemical energy has gained significant attention for understanding the processes of solar cells, photosynthesis and photocatalysis. Herein, inspired by the principles of light-addressable potentiometric sensor (LAPS) ${ }^{1}$ and lightactivated electrochemistry (LAE) ${ }^{2}$, a frequency responsive photoelectric system is proposed using modulated light to irradiate on a semiconductor-electrolyte structure.

\section{Methods}

In this system, double-polished silicon (100) substrates (boron doped, 1-10 $\Omega \mathrm{cm}$ ) were adopted as the research model. A diode laser $(405 \mathrm{~nm})$ modulated electronically $(1 \mathrm{kHz})$ was used for AC photocurrent excitation. Photocurrent measurements were carried out in $10 \mathrm{mM}$ phosphate buffer in an electrochemical cell consisting of a platinum electrode and an $\mathrm{Ag} / \mathrm{AgCl}$ electrode as the counter and reference electrodes, respectively.
AC photocurrents were measured by a lock-in amplifier while DC photocurrents were detected by a potentiostat (Fig 1).

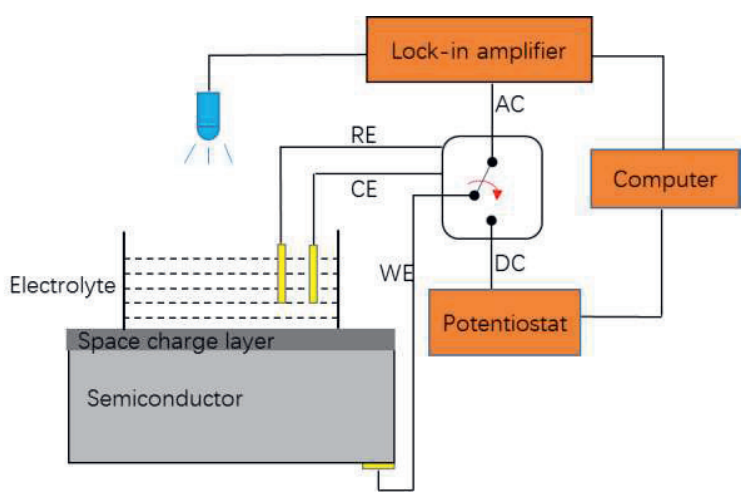

Fig. 1. Experimental set-up for $A C$ and $D C$ photocurrent measurements.

\section{Results and discussion}

The results show that under the same bias voltage and laser intensity, the signal-to-noise ratio (SNR) of the produced AC photocurrent was significantly higher than that of DC output (e.g. 946 vs. 4.6 at $0.7 \mathrm{~V}$, see Figs $2 \mathrm{a}$ and $2 \mathrm{~b}$ ). Furthermore, the silicon substrate shows a pH 
sensitivity of about $36 \mathrm{mV} / \mathrm{pH}$ (see Fig 2c), which is similar to the traditional silicon-based field-effect sensors such as LAPS with a $\mathrm{SiO}_{2}$ insulator. On the other hand, due to the absence of insulator, the photocurrent-voltage curve was steeper and displayed a greater photocurrent resulting in a higher sensitivity of photocurrent measurements compared to the silicon substrates with oxide ${ }^{3}$. The AC photocurrent kept stable in $15 \mathrm{~min}$ at bias voltage of $0.7 \mathrm{~V}$. The proposed simple and lowcost structure can not only be used for photoelectrochemical response measurements of different semiconductors with high SNR, but also be applied for the sensing of ions, DNA or living cells.

\section{Acknowledgements}

This work was supported by the National Natural Science Foundation of China (Grant No. 31470956, 31661143030), the Doctoral Fund of Education Ministry of China (Grant No.
2017M613146) and the Fundamental Research Funds for the Central Universities.

\section{References}

[1] J Wang, Y Zhou et al., High-sensitivity lightaddressable potentiometric sensors using silicon on sapphire functionalized with selfassembled organic monolayers, Sensors and Actuators B, 2015, 209: 230-236, DOI: 10.1016/j.snb.2014.11.071.

[2] Y Yang, S Ciampi et al., Light-Activated Electrochemistry for the Two-Dimensional Interrogation of Electroactive Regions on a Monolithic Surface with Dramatically Improved Spatial Resolution, J. Phys. Chem. C, 2016, 120, 13032-38, DOI: 10.1021/acs.jpcc.6b02289.

[3] L Du, C Wu, et al., Bioengineered olfactory sensor neuron-based biosensor for specific odorant detection. Biosensors and Bioelectronics, 2013, 40(1): 401-406, DOI: 10.1016/j.bios.2012.08.035

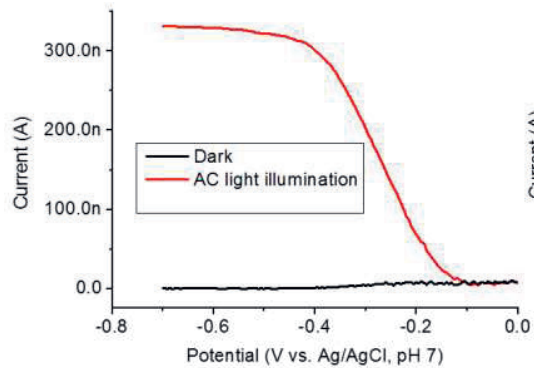

(a)

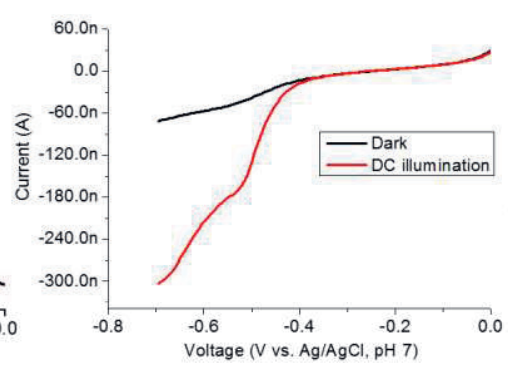

(b)

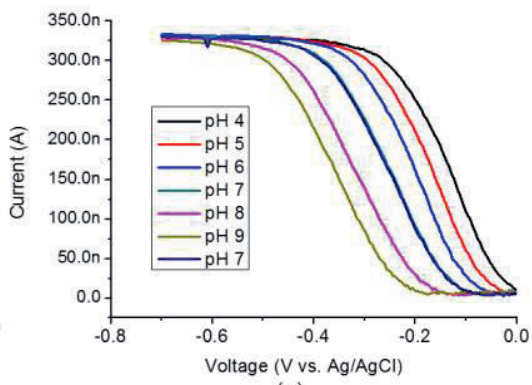

(c)

Fig. 2. Current-voltage curves of a p-type silicon substrate under $405 \mathrm{~nm}$ laser (red) and dark (black) measured in PBS at pH 7 (a) AC output measured with lock-in amplifier and (b) DC output measured with potentiostat; (c) AC photocurrent-voltage curves at different $\mathrm{pH}$ values. For AC photocurrent measurements, the laser was electronically modulated at $1 \mathrm{kHz}$. 